\title{
Evo-devo focus issue
}

\section{Heredity (2006) 97, 137-138. doi:10.1038/sj.hdy.6800878}

This issue features papers in evolutionary developmental biology - 'evo-devo' - that explore the roles of genetic variation in the evolution of developmental processes in animals. Development is central to evolution because the processes of development translate genotypes into organisms. Differences in gene function during development generate the variations in phenotype that can then be sieved by natural selection to yield adaptive evolution. Thus, if we are fully to understand the genetic basis of morphological evolution, we need to incorporate research on the mechanisms of development. This collection of papers reflects the way in which the field of evo-devo is expanding to consider genetic variation in natural populations, and is doing so in the context of the evolution of morphologies that are relevant in an ecological arena, such as in interactions with competitors, with potential mating partners or with predators.

Developmental biology has flourished over the past few decades, largely through the application of molecular genetics. The induction and manipulation of major mutations has identified genes and gene product functions that are central to development. Furthermore, it is now clear from comparative studies that the basic mechanisms of embryonic development are extremely ancient and have been highly conserved - to a surprising and exciting extent the same 'toolkit' of transcription factor families, intercellular signalling pathways and so on, is used to build highly divergent adult body forms such as flies, nematode worms, fish and mice. Evo-devo has slowly been extending this work from a few model organisms, to examine the more subtle morphological differences found among closely related species, and to determine whether genes involved here also contribute to differences between individuals within a species. Thus, a key issue is the extent to which genes with central developmental functions may harbor variation that is segregating within natural populations, providing the developmental basis of phenotypic variation. The papers in this issue show how some of the current work in evo-devo is beginning to address such fundamental issues in evolutionary genetics.

It is becoming clear that much of the evolution of morphological diversity has not occurred by appearance of novel genes, or by changes in gene number or in the function of a gene product. Instead, tinkering in a gene's complex regulatory apparatus can change the position or timing of gene expression in the embryo - teaching an old gene new tricks and altering the phenotype. The paper of Wittkopp (2006) discusses this crucial topic of the evolution of gene regulation.

Several of the papers in this issue illustrate the advantages of studying trait diversity among species that are closely related to one of the model organisms of developmental biology. Thus, Simpson and Marcellini (2006) review work on sensory bristle patterning in dipteran flies, and Parichy (2006) and Hoekstra (2006) discuss pigmentation patterns in Danio fishes and in species of mice, respectively. The Danio research demonstrates the additional power that comes from an ability to hybridize closely related species (eg enabling genetic complementation tests involving a 'candidate' mutation in the model species). Several other papers show how an understanding of development of the model organisms can provide an entry into examination of related groups that are more amenable in the wild and have fascinating field biology and evolutionary history. Thus, the work on Heliconius butterfly wing patterns (Joron et al, 2006), on dung beetle horns (reviews by Emlen et al (2006) and by Moczek (2006)), and on aphid wings (Braendle et al, 2006) is founded on knowledge and experimental tools derived from Drosophila, and is now deepening our understanding of the evolution of mimicry rings, of novel morphologies and of reproduction/dispersal polymorphisms. Similarly, the research on head and jaw morphologies in cichlid fish (Albertson and Kocher, 2006) is extending work on 'lab vertebrates' to a spectacular example of rapid speciation and parallel adaptive radiation.

One vigorous aspect of evo-devo is that due to employment of increasingly powerful methods for identifying developmentally significant genes and manipulating their function in non-model organisms, the field is rapidly expanding to include many systems which now lend themselves to studies both in the wild and in the laboratory. We have not been able to include many of these case studies - stalk-eyed flies, nematode worms, centipedes, stickleback fish and several plant systems all spring to mind - nevertheless this focus issue does represent a wide coverage of various animal groups, from invertebrates through to mice. This issue also demonstrates that evo-devo is effectively extending its scope into understanding the developmental changes that underlie the evolution of diverse morphologies at all biological levels. The papers range widely, from body plans (Swalla, 2006) and larval stages (Raff and Byrne, 2006), through to skeletal forms and surface patterns of bristles and pigmentation. Also, studies involving a major component of developmental plasticity are represented, notably in the papers on aphid wing dimorphism and on dung beetle horns, where plasticity and genetic polymorphism both contribute to the patterns of morphological variation found in nature.

Evo-devo is making great progress and future studies should continue to reveal how genetic change in the processes of development can lead to the abundant diversity in form that we observe in nature from major differences in body plan across the phyla, to the origins of key morphological innovations, 
through to variation among the individuals within populations.

PM Brakefield and V French Editors

E-mails: p.m.brakefield@biology.leidenuniv.nl; vernon.french@ed.ac.uk

\section{References}

Albertson RC, Kocher TD (2006). Heredity 97: 211-221.

Braendle C, Davis GK, Brisson JA, Stern DL (2006). Heredity 97: 192-199.
Emlen DJ, Szafran Q, Corley LS, Dworkin I (2006). Heredity 97: 179-191.

Hoekstra HE (2006). Heredity 97: 222-234.

Joron M, Jiggins CD, Papanicolaou A, McMillan WO (2006). Heredity 97: 157-167.

Moczek AP (2006). Heredity 97: 168-178.

Parichy DM (2006). Heredity 97: 200-210.

Raff RA, Byrne M (2006). Heredity 97: 244-252.

Simpson P, Marcellini S (2006). Heredity 97: 235-243.

Swalla BJ (2006). Heredity 97: 148-156.

Wittkopp PJ (2006). Heredity 97: 139-147. 